\title{
Molecular Cloning of a Novel Glutelin cDNA from Rice Seeds
}

\author{
Norihiro MITSUKAWA*, Hirofumi HAYASHI, Kayo YAMAMOTO, \\ Kunitomo KIDZU, Ryoichi KONISHI, Takehiro MASUMURA and Kunisuke TANAKA** \\ Laboratory of Genetic Engineering, Faculty of Agriculture, \\ Kyoto Prefectural University \\ Shimogamo, Kyoto 606-8522, Japan
}

Received 18 July 1998; accepted 14 August 1998

\begin{abstract}
A novel glutelin gene was cloned from a cDNA library of maturing rice seeds (Oryza sativa L. cv Nipponbare). The $2.0 \mathrm{kbp}$ insert contained an open reading frame encoding a 510 amino acid polypeptide $\left(\mathrm{M}_{\mathrm{r}}\right.$ 57,116). This novel glutelin shares 46 to $49 \%$ amino acid identity with previously identified rice glutelins. A phylogenetic analysis of cloned glutelins indicates that this gene constitutes a new, fifth class of glutelin gene families. The Asn-Gly processing sequence which is highly conserved in 11S seed storage proteins is replaced by Asn-Val in the sequence of the novel glutelin. The amino acid composition extending 50 residues both upstream and downstream of the Asn-Val site was less hydrophilic than in other glutelins. The $\mathrm{N}$-terminal half corresponding to the acidic domains of other glutelins possesses a higher $\mathrm{pI}$ value (7.46) than found in other glutelins. Expression of the gene was detected in maturing seeds, but not in roots or leaves.
\end{abstract}

\section{Introduction}

Rice glutelins share amino acid sequence homology with the $11 \mathrm{~S}$ globulins of legume species, such as soybean glycinin [1] and pea legumin [2], but differ from other globulins in their insolubility in saline solutions. The glutelins are synthesized as preproglutelin ( $c a .59 \mathrm{kDa}$ ) by endoplasmic reticulum (ER) bounded polysomes. Processing from prepro- to pro -glutelin $(c a .57 \mathrm{kDa})$ occurs co-translationally. The precursors are then transported via the Golgi complex to the vacuole, and are proteolyticaly cleaved to generate acidic $(37-39 \mathrm{kDa})$ and basic subunits $(22-23$ $\mathrm{kDa})[3,4]$. During seed maturation, glutelins accumulate in type II protein bodies (PB-II) which derive from the vacuole [5], and the glutelin subunits assemble into polymers through inter-molecular disulfide bond formation [6].

An Asn-Gly dipeptide is conserved as the post-translational cleavage site in $11 \mathrm{~S}$ legumin-like storage proteins from dicotyledon and monocotyledon plants. Proteolytic enzymes which cleave these storage proteins were purified from pumpkin $[7]$, soybean $[8,9]$ and rice [10]. Asparagine-specific cysteine proteases can cleave the Asn-Gly peptide bond. In rice seed, there is an unprocessed pro-glutelin which reacts both

\footnotetext{
* Present address : Mitsui Plant Biotechnology Research Institute TCI-D21, Sengen 2-1-6, Tsukuba, Ibaraki 305-0047, Japan.

** To whom correspondence should be addressed
}

with anti-acidic and anti-basic antibody [11]. This pro-glutelin also accumulates in PB-II [5]. There is no experimental evidence to indicate whether proglutelin is an unprocessed form of a known glutelin or an unprocessed glutelin homologue.

A number of glutelin cDNAs and genomic clones were isolated from rice [12-21]. These cDNAs and genomic clones are divided into at least four subfamilies based on their amino acid sequence homology. Takaiwa et al. [13] isolated two classes of glutelin cDNAs (Type I ; pREE61 and pREE103, Type II; pREE77 and pREE99) which share 95\% identity with each other and belong to the first subfamily. Okita et al. [19] reported a second subfamily of glutelin cDNAs (pG22) and genomic clones (Gt3). Two cDNA sequences in a third subfamily were reported by Masumura et al. [18] ( $\lambda$ RG21) and Takaiwa et al. [14] (pREEK1). A gene representing a fourth subfamily was reported by Takaiwa et al. [15] (GulB -1 , GulB-2 and GulB-3).

In this study, we have cloned a novel glutelin cDNA and showed that this cDNA belongs to a fifth subfamily of rice seed glutelins. The amino acid sequence deduced from this cDNA shares $40-45 \%$ homology with glutelin sequences of other classes. Instead of the Asn-Gly processing site conserved among all previously reported glutelins, this cDNA encodes Asn-Val. We discuss the possible ramifications on processing of the novel glutelins in vacuoles. 


\section{Materials and methods}

\section{1 Isolation of a novel glutelin cDNA from a rice seed library}

To obtain candidate clones for seed storage proteins, a $\lambda$ gt11 cDNA library from developing rice seeds [22] was screened with single stranded cDNA probes prepared from poly $(\mathrm{A}){ }^{+}$RNAs of developing rice seeds. Total RNAs were isolated from developing seeds (10 and 22 days after flowering; DAF) by the SDS-phenol method [23]. Poly (A)+RNAs were purified from the total RNAs by retention on oligo (dT) -cellulose columns [24], and these were reversetranscribed in the presence of ${ }^{32} \mathrm{P}-\mathrm{dATP}$ by $\mathrm{RAV}-2$ reverse transcriptase (TAKARA) to generate single stranded cDNA probe. Using Hybond-N nylon membranes (Amersham), duplicate plaque lifts were prepared from the library plated out on LB plates. These were hybridized with the cDNA probe in $6 \times$ SSC solution containing $50 \%$ formamide, $0.1 \%$ SDS, $5 \times$ Denhardt's solution, and $200 \mu \mathrm{g} / \mathrm{m} l$ denatured salmon sperm DNA for 16 hours at $37^{\circ} \mathrm{C}$. The membranes were washed twice with $6 \times$ SSC solution containing $0.1 \% \mathrm{SDS}$ at $25^{\circ} \mathrm{C}$ for $45 \mathrm{~min}$.

To select novel storage protein cDNAs, phage plaques of the candidate clones were transferred to membranes and then hybridized with a mixture of previously isolated rice storage protein cDNA probes ; $\lambda \mathrm{RP10}$ (10 kDa prolamin) [25], $\lambda \mathrm{RM} 7$ (13 kDa prolamin) [22], $\lambda$ RM4 (13 kDa prolamin) [Mitsukawa et al. in preparation], $\lambda$ RG1 (Type I glutelin) [Masumura et al. unpublished] (identical to pREE61 [13]). The membranes were washed with $1 \times \mathrm{SSC}$ solution containing $0.1 \% \mathrm{SDS}$ at $25^{\circ} \mathrm{C}$ twice for 45 min. Insert fragments of the non-hybridizing clones were subcloned into the EcoR I site of pBluescript $\mathrm{KS}^{+}$(Stratagene). End sequences of cDNA inserts were determined, and compared with previously reported nucleotide sequences of rice storage proteins. Full-length cDNA clones were isolated by rescreening using a novel clone as probe.

\section{2 DNA sequencing and analysis}

Nucleotide sequencing of the cloned cDNAs was performed using the Taq Dye Primer Cycle Sequencing kit in combination with a 373A DNA sequencer (Applied Biosystems). Nucleotide and amino acid sequence was analyzed using the sequence analysis programs MacVector (Eastman Kodak) and the signal peptide prediction program, Signalp Ver. 1. 0 [26]. Database searches were performed with the NCBI BLAST algorithm search program [27]. The amino acid sequences were aligned using the multiple align- ment program CLUSTAL W [28], and based on this alignment a phylogenic analysis of the rice glutelins was carried out by the neighbor-joining method [29] and a phylogenic tree was drawn using the software TREEVIEW [30].

\section{3 Northern and Southern blot analyses}

Total RNA was prepared from developing seeds 7 , 14 and 21 DAF, and from mature seeds, roots, leaves and etiolated leaves by the SDS-phenol method [23]. Aliquots $(5 \mu \mathrm{g})$ of each total RNA were fractionated on an $1.0 \%(\mathrm{w} / \mathrm{v})$ agarose gel containing formaldehyde, then blotted onto Hybond-N+ membranes (Amersham). Filters were hybridized with ${ }^{32} \mathrm{P}-$ labeled cDNA insert of $\lambda$ RG55 clone in $50 \%$ formamide, $6 \times$ SSPE, $5 \times$ Denhardt's solution, $0.5 \%$ SDS and $100 \mu \mathrm{g} / \mathrm{m} l$ salmon sperm DNA at $42^{\circ} \mathrm{C}$ overnight, and washed with $0.1 \times \mathrm{SSC}$ and $0.1 \% \mathrm{SDS}$ at $65^{\circ} \mathrm{C}$.

For Southern blotting, genomic DNA was prepared from rice leaves as described [31] then digested with restriction enzymes (Bam H I , EcoR I , EcoRV and Hind III). These DNA fragments were separated on a $0.8 \%$ agarose gel and transferred onto a nylon membrane (Hybond-N+, Amersham). Filter was hybridized with ${ }^{32} \mathrm{P}$-labeled cDNA insert of $\lambda$ RG55 clone in $6 \times \mathrm{SSPE}, 5 \times$ Denhardt's solution, $0.5 \% \mathrm{SDS}$ and $100 \mu \mathrm{g} / \mathrm{m} l$ salmon sperm DNA at $65^{\circ} \mathrm{C}$ overnight, and washed with $0.1 \times \mathrm{SSC}$ and $0.1 \% \mathrm{SDS}$ at $65^{\circ} \mathrm{C}$

\section{Results}

\section{1 Isolation and characterization of a novel glutelin cDNA clone}

A glutelin cDNA clone was isolated from a cDNA library prepared from maturing rice seeds. To obtain candidate storage protein cDNAs, we selected clones which were differentially expressed at a late stage of seed development. The cDNA library was screened using single strand cDNAs prepared from 10 and 22 DAF poly (A) ${ }^{+}$RNAs. Out of $8 \times 10^{4}$ recombinant plaques, forty clones showed increased signal intensity when hybridized with the 22 DAF poly (A) RNA probe. In order to distinguish novel storage protein clones from known ones, 20 of these clones were hybridized with a probe comprising a mixture of previously isolated storage protein cDNAs encoding $10 \mathrm{kDa}$ prolamin, $13 \mathrm{kDa}$ prolamin or Type I glutelin. Fourteen clones didn't hybridize with these probes. These clones were subcloned into the pBluescript $\mathrm{KS}^{+}$ plasmid vector and sequenced. Searches of protein database using the NCBI BLAST algorithm revealed 
ATTCTTCCATTGTGCCTTGGCCTCCTTCTCTTCTTCCAAGTGTCCATGGCACAATTTTCATTTGGGGGAAGCCCACTTCAGAGCCCACGTGGATTTAGGGGAGACCAAGATAGTCGTCA

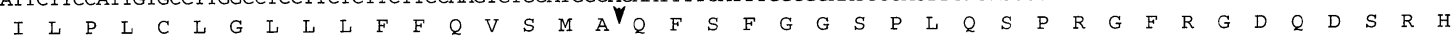
CAATGTCGTTTTGAGCACCTCACCGCCCTTGAGGCAACACACCAGCAGAGATCTGAAGCTGGATTCACTGAGTACTACAACATTGAGGCAAGAAATGAGTTCCGTTGTGCCGGAGTGAGC

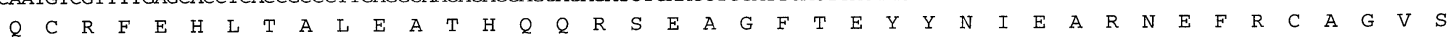
GTGAGGCGCTTAGTCGTCGAGAGCAAGGGCTTAGTTTTACCAATGTATGCTAATGCTCACAAGCTTGTCTACATCGTCCAAGGTCGGGGAGTGTTTGGGATGGCACTGCCTGGTTGTCCA

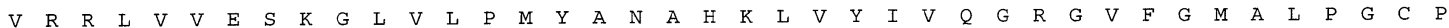
GAGACGTTCCAGTCAGTTAGGTCTCCCTTTGAGCAAGAGGTGGCAACAGCTGGTGAGGCTCAATCATCAATCCAAAAAATGAGAGACGAGCACCAGCAACTTCACCAATTCCACCAAGG'

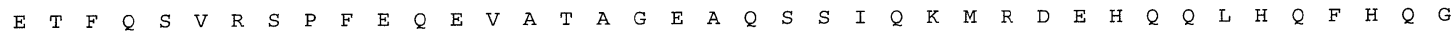
GATGTAATCGCAGTGCCAGCTGGAGTAGCCCACTGGCTATATAACAATGGTGATTCTCCTGTGGTTGCTTTCACTGTCATCGACACCAGCAACAATGCCAACCAGCTCGATCCTAAAAGA

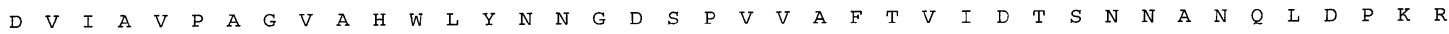
AGGGAGTTTTTCTTGGCTGGAAAGCCTAGAAGTAGCTGGCAGCAGCAATCGTACTCATACCAGACAGAACAACTGAGCAGAAATCAGAACATCTTTGCTGGGTTCAGCCCAGATTTACTT $\begin{array}{llllllllllllllllllllllllllllllllllllllllllllllllllll}R & E & F & F & L & A & G & K & P & R & S & S & W & Q & Q & Q & S & Y & S & Y & Q & T & E & Q & L & S & R & N & Q & N & I & F & A & G & F & S & P & D & L & L\end{array}$ TCTGAAGCCCTGAGTGTGAGCAAGCAAACTGTGTTGAGGCTCCAAGGCCTGAGTGACCCAAGAGGTGCCATCATTAGAGTTGAAAATGGGCTCCAGGCACTGCAGCCCTCTCTCCAAGT

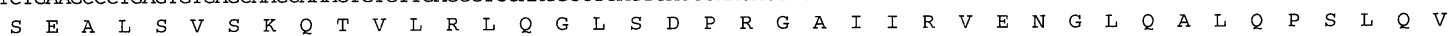
GAGCCAGTGAAAGAGGAACAAACCCAAGCTTACTTGCCAACCAAGCAGCTACAGCCCACCTGGTTGCGAAGTGGTGGAGCTTGCGGCCAGCAAAATGTCCTAGATGAAATTATGTGTGCA

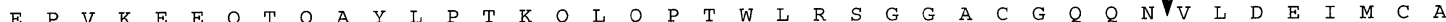
TTTAAGTTGAGGAAGAACATAGACAACCCACAATCCAGTGACATATTTAACCCCCATGGTGGAAGGATCACAAGGGCCAATAGCCAGAATTTCCCAATACTCAATATCATCCAGATGAGT

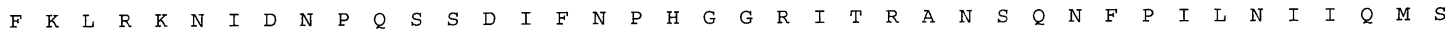
GCCACCAGAATCGTTCTCCAAAATAATGCCTTGCTTACTCCTCATTGGACGGTAAACGCACACACGGTGATGTACGTGACCGCTGGCCAAGGGCACATCCAGGTGGTGGATCACCGTGGT

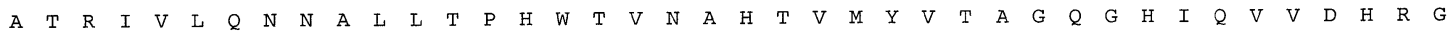
AGGAGTGTCTTTGATGGTGAGCTTCACCAACAGCAGATCTTGTTGATCCCACAGAACTTTGGAGTGGTGGTGAAGGCTCGACGTGAAGGATTTGCATGGGTATCCTTCAAGACCAATCAC

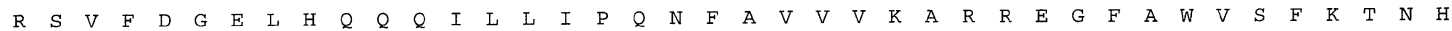
AATGCTGTCGACAGTCAGATCGCAGGGAAGGCCTCCATTCTTCGTGCTCTACCCGTTGACGTGGTCGCCAATGCTTATAGGCTTTCAAGGGAGGACTCTAGGCATGTAAAGTTCAACCGC

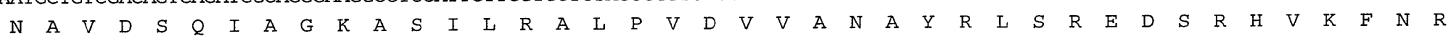
GGCGATGAGATGGCTGTCTTTGCTCCGAGGCGTGGGCCGCAACAGTATGCTGAGTGGCAGATCAACGAGAAGTAAACTAAATGTGTAACGATCTTACTGTAATGAATAATGTGAAGAAGA

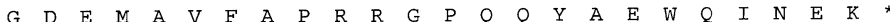
TTGCCTACACCTCTTTTTCCATAAAAATATCAATAAGCAATTACTAAGAACATGATAATACTCTCA

Fig. 1 Nucleotide and deduced amino acid sequence of the novel rice glutelin cDNA clone ( $\lambda$ RG55).

The arrowhead indicates the cleavage site of the predicted signal peptide. An arrow indicates the putative processing site of cystein protease.

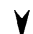

גRG55 MASMS-TILPLCLGLLIFFQVSMAQ--FSFGGSPLSPRGFREDQDSRHQCRFEHLTALEATHQQRSEAGFTEYYNIEARNEFRCAGVSVRRLVVESKGLVLPMY PREE61 MASINRPIVFFTVCLFLLCNGS LAOOLLGQSTSOWOSSR--RG---SPRECRFDRLQAFEPIRSVRSOAGTTEFFDVSNE- QFOCTGVSVVRRVIERRGLLLPHY

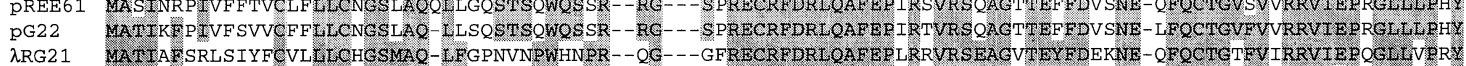

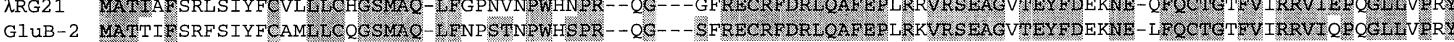

$\lambda$ RG55 ANAHKLVY TVGRGVFGMALPGCPETFQSVRSPFEQEVATAGEAOSS IQKMRDEHQQLHQFHQGDVIAVPAGVAHWL WNNGDSPVVAFTVIDTSNNANOLDRKRR PREE61 TNGASEVIIIQGRGITOPTFPGCPESYQQQFOQSGQAQLTESOSOS--QKFKDEHORTHRFROGDVIALPAGVAHWC YNDGEVVVUAT TVTDLNNGANOLDPROR

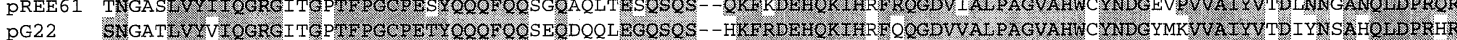

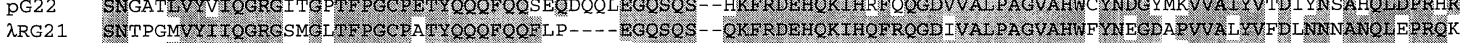

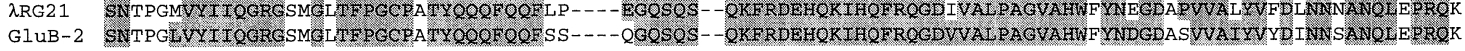

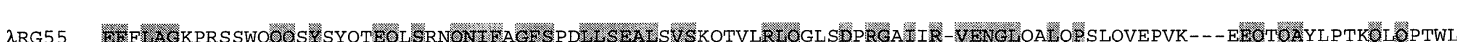
PREE61 DFLLA GN--KRNPQAYRREVEERS--QNIFSGESTELLSEALCVSGQVAROLOCONDORGETVR-VEHGLSLLOPYASLQEQE-QGQVQSRERYQEGOYQQSQY 300

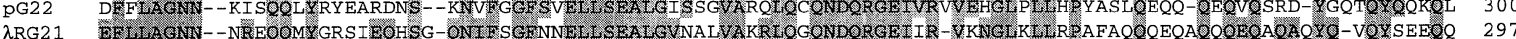

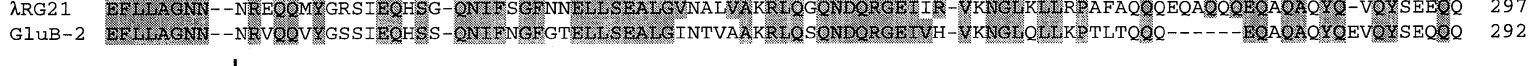

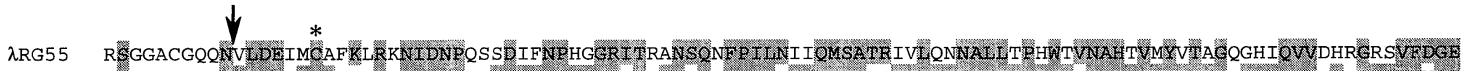

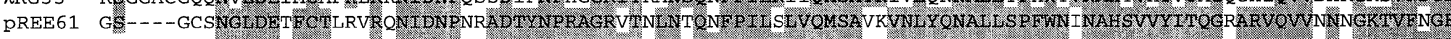
PG22 QG----SCSNCLDETFCTMRVR QNIDNPNLADTYNPRAGRTTYLNGQKFPTLNLVOMSAVKVNLY ONAL VSPFWNI NAHSVVYITOGRARVOVVNNNGKTVFDGE

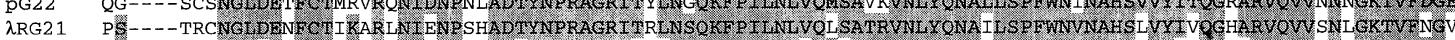

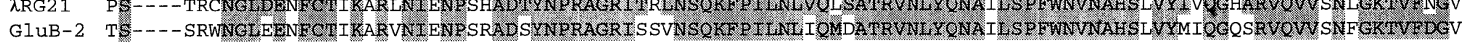

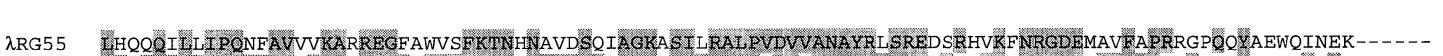

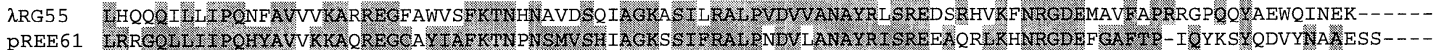

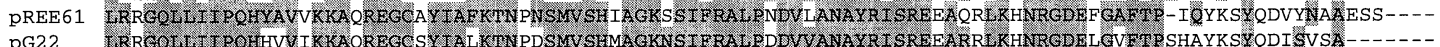

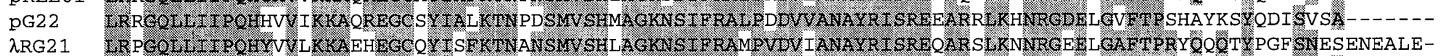

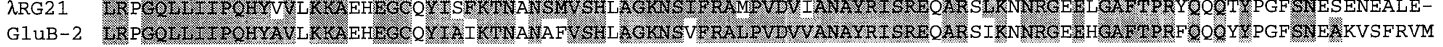

Fig. 2 Comparison of amino acid sequences drived from the novel rice glutelin cDNA ( $\lambda$ RG55) with following rice prepro-glutelin sequences (locus GenBank accession numbers of the sequences are indicated in parentheses) : pREE61 (OSGLUI1, X05661) [13], pG22 (RICGT22A, M28159) [19], $\lambda$ RG21 (OSGLUT21, X14393) [18], GluB-2 (OSGLUTB, X54192) [15]. Identical amino acid residues are represented by shaded boxes. Dashes indicate gaps in the amino acid sequences used to optimally align the sequences. The arrowhead and arrow indicate the predicted cleavage site of signal peptidase and the putative processing site of cysteine protease, respectively. The asterisks represent the cysteine residues which may be involved in disulfide linkages. 
that one cDNA clone, $\lambda \mathrm{RG} 5$, was homologous with rice glutelin genes. Using this cDNA clone as a probe, a full-length cDNA clone, $\lambda$ RG55 [46] was isolated.

Figure 1 shows the nucleotide and deduced amino acid sequence of the isolated cDNA clone $\lambda$ RG55. We used the nucleotide sequence as a query to search the GenBank expressed sequence tag (EST) data base and identified 41 EST entries that were nearly identical to the novel glutelin sequence. The EST clone, 96AS0372 [Nahm et al. unpublished, GenBank accession number AA751633] showed highest search score among the entries. The open reading frame of $\lambda$ RG55 encodes a polypeptide of 510 amino acid residues. The calculated molecular mass of the precursor (prepro-glutelin) is 57,116 . The protein lacks $\mathrm{N}^{-}$ glycosylation sites but possesses four cysteine residues which are conserved in other rice glutelins [16] (Fig. 2). Using the signal peptide prediction program Signalp Ver.1. 0, the signal peptide sequence of the prepro-glutelin was predicted to comprise Met1 through Ala23. The molecular mass of the predicted

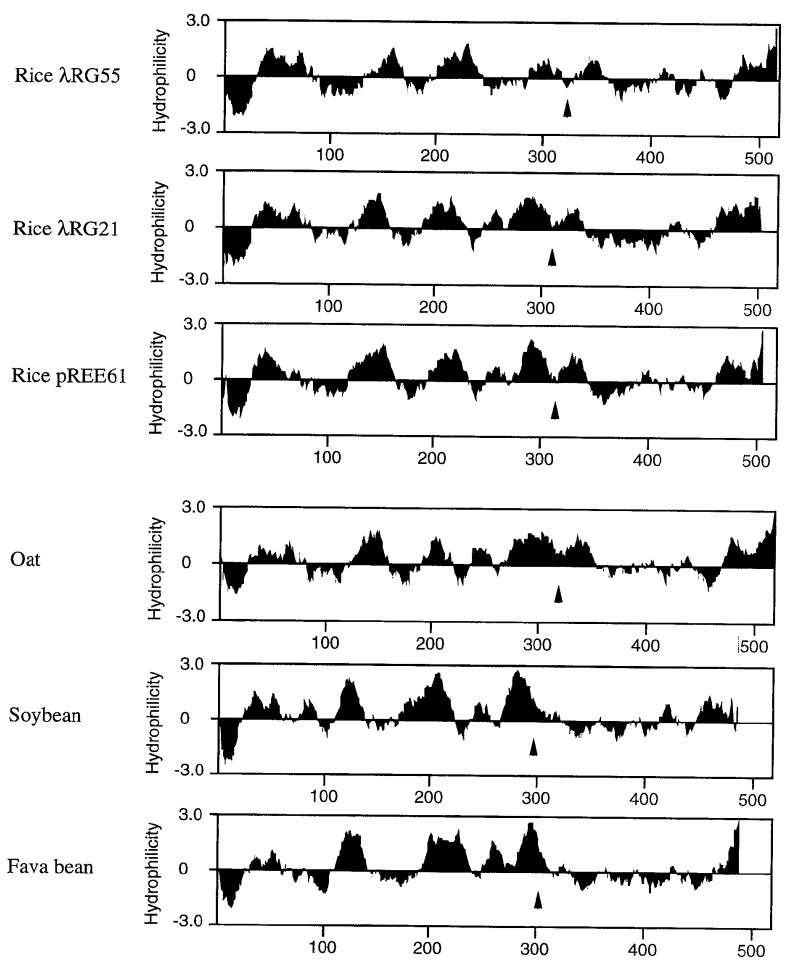

Fig. 3 Hydrophilicity profiles of the novel rice glutelin ( $\lambda$ RG55) compared with reported glutelin $\lambda$ RG21 (OSGLUT21, X14393) [18], pREE61 (OSGLUI1, X05661) [13], oat 11S globulin (ASTSPGLBA, M21405) [42], soybean glycinin (GMGY3, X15123) [43] and fava bean legumin (VFCBE4, X03677) [44]. Hydrophilicity values for a window of 21 residues were calculated by MacVector using algorithms of Kyte and Doolittle [45]. Arrowheads refer to putative post-translational processing sites. pro-glutelin is therefore 54,784. Although all other pro-glutelins have an Asn-Gly sequence which is also conserved in the $11 \mathrm{~S}$ globulins of soybean [1] and serves as a post-translational proteolytic cleavage site, the novel glutelin has Asn318-Val319 in a corresponding position. A hydrophilicity plot analysis indicated that the pattern of $\lambda$ RG55 is similar to those of other glutelins (Fig. 3). This result suggests that the protein encoded by $\lambda$ RG55 may belong to glutelin. The analysis shows the region extending about 50 residues both upstream and downstream of the AsnVal site was less hydrophilic than in other glutelins.

(Fig. 3). Interestingly, the $\mathrm{N}$-terminal half (amino acids 24 to 318 ) that corresponds to the acidic subunits of the other glutelins has a much higher calculated pI value (7.46) than in most other glutelins ( $\mathrm{pI} 6.70$ to $7.36)$.

\section{2 Novel glutelin cDNA constitutes a new subfamily of rice glutelin genes}

Amino acid sequences of the novel and previously cloned glutelins were analyzed using the sequence analysis software MacVector (Eastman Kodak), as well as a multiple sequence alignment program, CLUSTAL W [28], and a phylogenic tree drawing program, TREEVIEW [30]. The novel glutelin gene constitutes a fifth class of rice glutelin genes. A

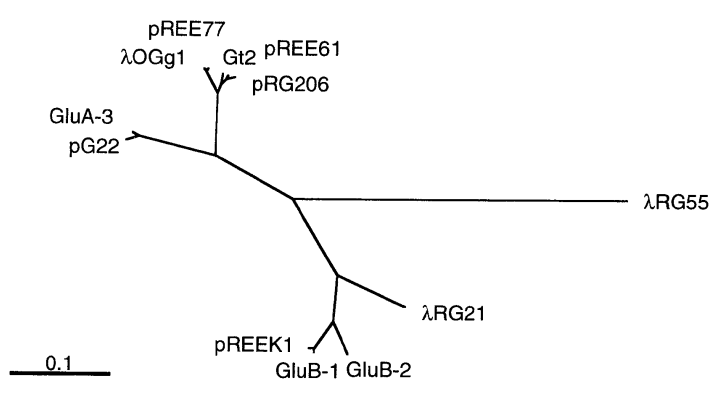

Fig. 4 Unrooted phylogenic tree inferred from amino acid sequences of rice prepro-glutelin.

The phylogenic tree was constructed with the deduced amino acid sequences from following sequences: $\lambda$ RG55 (this study), pREE61 (OSGLUI1, X05661) [13], pREE77 (OSGLUII1, X05663) [13], pRG206 (RICGLUTA, M17513) [16], Gt2 (RICGT2A, L36819) [19], pG22 (RICGT22A, M28159) [19], $\lambda$ OGg1 (RICEGLUTE, D00584) [21], GluA-3 (OSGLUA3, X54313) [12], $\lambda$ RG21 (OSGLUT21, X14393) [18], pREEK1 (OSGLUT, X14568) [14], GluB-1 (OSGLUB1, X54314) [12], GluB -2 (OSGLUB2, X54192) [12]. The phylogenic tree was generated using CLUSTAL W [28] and TREEVIEW [30] programs. The scale bar indicates a divergence of 0.1 amino acid substitution per site. 
phylogenic tree of the glutelins is shown in Fig. 4. The tree shows that the glutelin genes fall into three well-separated clusters. Two of these clusters are divided in turn each into two smaller clusters. The novel glutelin shared the least amino acid sequence identity (46-49\%) with the other glutelins, although the amino acid sequence identity among the remaining glutelin families ranged between $63 \%$ and $84 \%$.

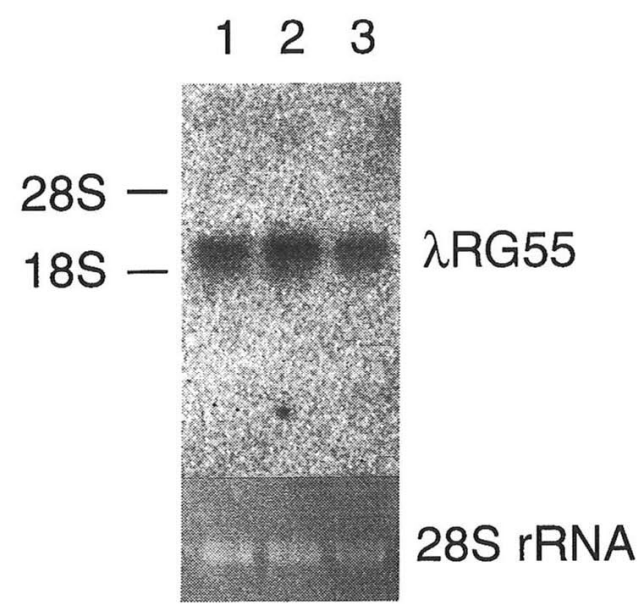

Fig. 5 Northern blot analysis of maturing rice seeds probed by the novel glutelin cDNA insert.

Total RNAs isolated from maturing seeds (7 DAF, lane 1; $14 \mathrm{DAF}$, lane 2; and $21 \mathrm{DAF}$, lane 3 ) were applied on a $1.0 \%$ denaturing agarose gel.

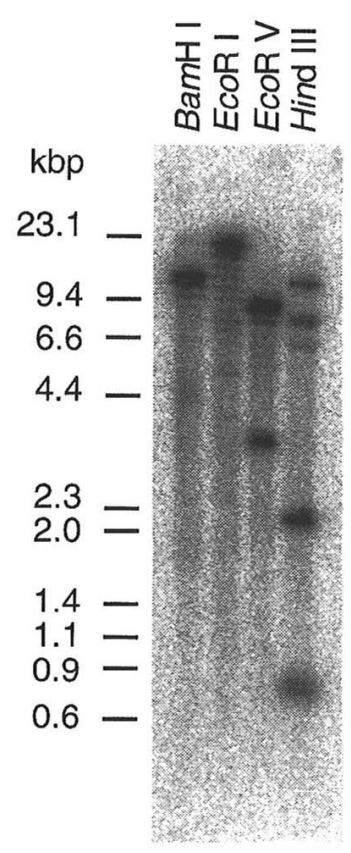

Fig. 6 Southern blot analysis of rice genomic DNA.

Aliquots of $2 \mu \mathrm{g}$ of rice germ DNA were digested with $B a m \mathrm{H} \mathrm{I}, E c o$ R I, EcoRV and Hind III. The fragments were resolved on a 0 . $8 \%$ agarose gel and probed with radiolabeled cDNA insert of $\lambda$ RG55.

\section{3 Gene expression pattern and genomic DNA analysis}

To determine the expression pattern of the novel glutelin gene, we performed Northern blot analyses by using total RNA isolated from various rice tissues. The novel glutelin gene was expressed exclusively in maturing seeds (Fig.5). No expression was seen in roots, leaves or etiolated leaves (data not shown). The transcript (about $2.0 \mathrm{~kb}$ ) in seeds appeared early during maturation ( 7 DAF). The amounts of the transcript increased at $14 \mathrm{DAF}$ and decreased at 21 DAF (Fig. 5). Expression of this gene thus correlates temporally with glutelin accumulation [32].

Genomic DNA was isolated from rice germ, and digested with BamH I, EcoR I , EcoRV and Hind III for Southern blot analysis (Fig.6). The $\lambda$ RG55 cDNA probe hybridized to a $14 \mathrm{kbp} B a m \mathrm{H} \mathrm{I}$ and a 25 kbp EcoR I fragment, while two or five bands were found after DNA digestion with EcoR V and Hind III. The result indicates the gene copy number per haploid genome is two or three, because the cDNA sequence have no $B a m \mathrm{H} \mathrm{I}, E c o \mathrm{R} \mathrm{I}$ and Eco RV sites, and two Hind III sites.

\section{Discussion}

The full-length cDNA reported here encodes a novel protein homologous with known rice glutelins. This novel glutelin retains the least amino acid sequence identity $(46-49 \%)$ of any glutelin reported to date. We assigned this glutelin to a fifth gene family based on amino acid sequence homology (Fig. 2) and phylogenic analysis (Fig. 4).

The novel glutelin does not have the Asn-Gly processing site highly conserved among 11S globulins, although rice glutelins belong to the $11 \mathrm{~S}$ globulin superfamily [16], and the conserved processing site is found in all glutelins previously reported. There is no data indicating whether the novel glutelin is cleaved by processing enzymes at the Asn318-Val319 site. However, variations on the Asn-Gly sequence have been reported for other legumin-like proteins isolated from pea (Asn-Phe) [33], ginkgo (Asn-Asn) [34] and Japanese red cedar (Asn-Phe) [35]. There is experimental evidence that the proteins of pea and ginkgo can be cleaved by the processing proteases on the C-terminal side of Asn. The purified soybean enzyme can cleave between the two Asn residues in the sequence $\mathrm{NH}_{2}-\mathrm{Gly}-\mathrm{Asn}-\mathrm{Asn}-\mathrm{Val}-\mathrm{Qln}-\mathrm{Qln}-\mathrm{Leu}-$ $\mathrm{COOH}$, but not between Asn and Val [34]. Concanavarin A, a vacuolar protein in jackbean seed cotyledons is cleaved between Asn148 and Val149 [36].

The amino acid sequences homologous to the novel 
glutelins have not been reported in previous protein sequencing analyses of glutelins. Acidic subunits of glutelins were separated into sixteen spots by two dimentional electrophoresis [37]. Hirano et al. [38] and Komatsu et al. [39] reported N-terminal sequences of eight major glutelin acidic subunits. However, none of these sequences match the amino acid sequence of the novel glutelin. One potential explanation is that the novel glutelin cannot be cleaved into basic and acidic subunits. Alternatively, this glutelin may compose only a minor component of rice glutelins.

This novel glutelin may assemble into protein bodies regardless of whether the precursors are processed or not. All four cysteine residues encoded by $\lambda$ RG55 are conserved among rice glutelins. Two of these residues, Cys125 and Cys324 are conserved in soybean glycinins. These residues may be involved in disulfide linkages, because corresponding residues in glycinins have been found to be involved in linking the acidic subunits of glycinins [40]. Synthesized in vitro, the uncleaved precursor of glycinin was able to self-assemble into trimers [41]. Still, we cannot exclude the possibility that the precursor of this novel glutelin is cleaved and incorporated into PB-II normally.

Further investigations will be needed to determine whether this glutelin can be cleaved into subunits by the maturing enzyme. The temporal expression pattern and seed-specificity of the gene characterized here, as well as the resemblance of the predicted protein product to known glutelins all indicate a high probability that this gene encodes a seed storage protein. Nevertheless, conclusive evidence on this point will require purification and partial amino acid sequencing of the protein from rice seeds.

\section{Acknowledgments}

We are grateful to Dr. Daisuke Shibata (Mitsui Plant Biotech. Res. Inst.) for stimulating discussion and suggestions. We are indebted to Dr. Robert F. Whittier (Amersham Pharmacia Biotech) for critical reading the manuscript. We thank Dr. Yumiko Shirano (Mitsui Plant Biotech. Res. Inst.) for sharing filters of leaves, etiolated leaves and roots RNAs. This research was supported by grants from the Ministry of Education, Science and Culture (Japan), and "Research for the Future" Program of the Japan Society for the Promotion of Science.

\section{References}

[1] Nielsen, N.C., Dickinson, C.D., Cho, T.-J., Thanh, V. H., Scallon, B. J., Fischer, R. L., Sims, T. L., Drews, G. N., Goldberg, R. B., 1989. Plant Cell, 1: 313-328.
[2 ] Lycett, G. W., Croy, R. R., Shirsat, A. H., Boulter, D., 1984. Nucl. Acids Res., 12: 4493 $-4506$.

[3 ] Yamagata, H., Tanaka, K., Kasai, Z., 1982. Agric. Biol. Chem., 46: 321-322.

[4] Luthe, D. S., 1983. Plant Sci. Lett., 32: 147158.

[ 5 ] Tanaka, K., Sugimoto, T., Ogawa, M., Kasai, Z., 1980. Agric. Biol. Chem., 44: 1633-1639.

[6] Sugimoto, T., Tanaka, K., Kasai, Z., 1986. Agric. Biol. Chem., 50: 3031-3035.

[ 7 ] Hara-Nishimura, I., Inoue, K., Nishimura, M., 1991. FEBS Lett., 294: 89-93.

[ 8 ] Scott, M. P., Jung, R., Muntz, K., Nielsen, N. C., 1992. Proc. Natl. Acad. Sci. USA, 89: 658-662.

[9] Muramatsu, M., Fukazawa, C., 1993. Eur. J. Biochem., 215: 123-132.

[10] Kato, H., Minamikawa, T., 1996. Eur. J. Biochem., 239: 310-316.

[11] Krishnan, H. B., Okita, T. W., 1986. Plant Physiol., 81: 748-753.

[12] Takaiwa, F., Kikuchi, S., Oono, K., 1986. FEBS Lett., 206: 33-35.

[13] Takaiwa, F., Kikuchi, S., Oono, K., 1987. Mol. Gen. Genet., 208: 15-22.

[14] Takaiwa, F., Kikuchi, S., Oono, K., 1989. Nucl. Acids Res., 17: 3289.

[15] Takaiwa, F., Oono, K., Wing, D., Kato, A., Plant Mol. Biol., 17: 875-885.

[16] Higuchi, W., Fukazawa, C., 1987., Gene, 55: 245 $-253$.

[17] Wang, C.-S., Shastri, K., Wen, L., Huang, J.-K., Sonthayanon, B., Muthukrishnan, S., Reeck, G. R., 1987. FEBS Lett., 222: 135-138.

[18] Masumura, T., Kidzu, K., Sugiyama, Y., Mitsukawa, N., Hibino, T., Tanaka, K., Fujii, S., 1989. Plant Mol. Biol., 12: 723-725.

[19] Okita, T. W., Hwang, Y.S., Hnilo, J., Kim, W. T., Aryan, A. P., Larson, R., Krishnan, H. B., 1989. J. Biol. Chem., 264: 12573-12581.

[20] Wen, L., Huang, J. K., Johnson, B. H., Reeck, G. R., 1989. Nucl. Acids Res., 17: 9490.

[21] Abe, K., Emori, Y., Kawasaki, H., Kondo, H., Suzuki, K., Arai, S., 1989. Agric. Biol. Chem., 53: 2969-2973.

[22] Masumura, T., Hibino, T., Kidzu, K., Mitsukawa, N., Tanaka, K., Fujii, S., 1990. Mol Gen. Genet., 221: 1-7.

[23] Yamagata, H., Tanaka, K., 1986. Plant Cell Physiol., 27: 135-145.

[24] Yamagata, H., Tamura, K., Tanaka, K., Kasai, Z., 1986. Plant Cell Physiol., 27: 1419-1422.

[25] Masumura, T., Shibata, D., Hibino, T., Kato, T., Kawabe, K., Takeba, G., Tanaka, K., Fujii, S., 1989. Plant Mol. Biol., 12: 123-130. 
[26] Nielsen, H., Engelbrecht, J., Brunak, S., von Heijne, G., 1997. Protein Eng., 10: 1-6.

[27] Altschl, S. F., Madden, T. L., Schäffer, A. A., Zhang, J., Zhang, Z., Miller, W., Lipman, D. J., 1997. Nucl. Acids Res., 25: 3389-3402.

[28] Thompson, J. D., Higgins, D. G., Gibson, T. J., 1994. Nucl. Acids Res., 22: 4678-4680.

[29] Saitou, N., Nei, M., 1987. Mol. Biol. Evol., 4: 406-425.

[30] Page, R. D. M., 1996. Comp. Appl. Biosci., 12: 357-358.

[31] Richards, E., 1987. In "Current protocols in molecular biology", (eds. by Ausubel, F. M., et al.), p. 2.3.1-2.3.3, Greene Publishing Associates and Wiley Interscience, New York.

[32] Yamagata, H., Sugimoto, T., Tanaka, K., Kasai, Z., 1982. Plant Physiol., 70: 1094-1100.

[33] March, J. F., Pappin, D. J. C., Casey, R., 1988. Biochem. J., 250: 911-915.

[34] Arahira, M., Fukazawa, C., 1994. Plant Mol. Biol., 25: 597-605.

[35] Wind, C., Häger, K.-P., 1996. FEBS Lett., 383 : 46-50.

[36] Carrington, D. M., Auffret, A., Hanke, D. E., 1985. Nature, 313: 64-67.
[37] Wen, T.-N., Luthe, D. S., 1985. Plant Physiol., 78: $172-177$.

[38] Hirano, H., Komatsu, S., Nakamura, A., Kikuchi, F., Kajiwara, H., Tsunasawa, S., Sakiyama, F., 1991. Theor. Appl. Genet., 83: 153-158.

[39] Komatsu, S., Kajiwara, H., Hirano, H., 1993. Theor. Appl. Genet., 86: 935-942.

[40] Staswick, P. E., Hermodson, M. A., Nielsen, N. C., 1984. J. Biol. Chem., 259: 13431-13435.

[41] Dickinson, C. D., Hussein, E. H. A., Nielsen, N. C., 1989. Plant Cell, 1: 459-469.

[42] Shotwell, M. A., Afonso, C. L., Davies, E., Chesnut, R. S., Larkins, B. A., 1988. Plant Physiol., 87: 698-704.

[43] Cho, T. J., Nielsen, N. C., 1989. Nucl. Acids Res., 17: 4388.

[44] Baumlein, H., Wobus, U., Pustell, J., Kafatos, F. C., 1986. Nucl. Acids Res., 14: 2707-2720.

[45] Kyte, J., Doolittle, R. F., 1982. J. Mol. Biol., 157: 105-132.

[46] The nucleotide sequence was submitted to DDBJ, EMBL and GenBank nucleotide sequence databases. The accession number of $\lambda$ RG55 is AB016501. 